\title{
Trastorno linfoproliferativo post trasplante renal en un niño
}

\author{
Sonia Márquez U ${ }^{1}$, María Soledad Peredo G ${ }^{1}$, \\ Andrea Vogel $\mathbf{S}^{1}$, Paulina Ibáñez $\mathbf{A}^{2}$. \\ Lymphoproliferative disorders \\ after renal transplantation in a child
}

\begin{abstract}
Post transplant lymphoproliferative disorder (PTLD) is the commonest form of post transplant malignancy in children. The incidence in renal transplant recipients varies between 2\%-4\%. They are characterized by uncontrolled B lymphocyte proliferation, in most cases driven by Epstein Barr virus (EBV). They are more common in younger children, EBV seronegative patients and those who receive aggressive immunosuppression. PTLD commonly presents in an unspecific form and it requires high suspicion rate for its diagnosis, especially in children with risk factors. We report a twelve yearold girl who developed fever, sore throat and lymph node enlargement, six months after receiving a renal allograft. Laboratory assessment and imaging studies were compatible with PTLD, which was confirmed by biopsy. Treatment was reduction of immunosuppression and surveillance. The patient had a favorable evolution (Rev Méd Chile 2009; 137: 405-10).
\end{abstract}

(Key words: Immunosuppression; Kidney transplantation; Lymphoproliferative disorders)

Recibido el 13 de marzo, 2008. Aceptado el 13 de agosto, 2008.

${ }^{1}$ Unidad de Nefrología Pediátrica, Departamento de Pediatría, Facultad de Medicina, Pontificia Universidad Católica de Chile. Santiago de Chile. ${ }^{2}$ Departamento de Anatomía Patológica, Facultad de Medicina, Pontificia Universidad Católica de Chile. Santiago de Chile.

$\mathrm{E}$

trasplante renal es el tratamiento de elección de la enfermedad renal crónica terminal (ERCT), mejorando la calidad de vida y sobrevida de los pacientes ${ }^{1,2}$. La inmunosupresión es fundamental para preservar el injerto, pero no está exenta de efectos adversos como el desarrollo de neoplasias $^{3}$. Los trastornos linfoproliferativos post trasplante (TLPT) constituyen la neoplasia post trasplante más frecuente en niños y una causa

Correspondencia a: Sonia Márquez Ulloa. Fax: 02-8843874. E mail: smarquez@uc.cl importante de morbimortalidad ${ }^{4}$. Se caracterizan por la proliferación descontrolada de linfocitos B, inducida generalmente por infección por virus Epstein Barr (VEB) ${ }^{5}$. Se observan más frecuentemente en niños menores, que no han presentado infección por VEB y que reciben inmunosupresión agresiva. La presentación clínica es inespecífica y su diagnóstico requiere un alto índice de sospecha, particularmente en pacientes con factores de riesgo conocidos. El diagnóstico definitivo se basa en los hallazgos histopatológicos ${ }^{6}$. Presentamos el caso de una niña con un trastorno linfoproliferativo post trasplante renal y una descripción de los aspectos fundamentales de esta patología. 


\section{Caso ClínICO}

Paciente de sexo femenino de 12 años de edad con antecedente de ERCT secundaria a glomeruloesclerosis focal y segmentaria, en diálisis peritoneal desde los 5 años. Recibe trasplante renal donante cadáver a los once años, sin incidentes. Serología del donante: IgG VEB (+) e IgG citomegalovirus(CMV) $(+)$; serología del receptor IgG e IgM VEB (-), IgG CMV (+). Recibe terapia de inducción con basiliximab (2 dosis de $10 \mathrm{mg}$ ) y metilprednisolona $\left(300 \mathrm{mg} / \mathrm{m}^{2}\right)$ y tratamiento de mantención con tacrolimus $(0,3 \mathrm{mg} / \mathrm{kg} / \mathrm{d})$, micofenolato mofetil (MMF) $\left(765 \mathrm{mg} / \mathrm{m}^{2} / \mathrm{d}\right)$ y prednisona $5 \mathrm{mg} / \mathrm{d}$, con niveles de tacrolimus adecuados (10,6 ng/ml). Recibe profilaxis de Pneumocystis jiroveci con trimetropim-sulfametoxazol y antifúngica con nistatina.

A los 6 meses post trasplante presenta cuadro de odinofagia y fiebre intermitente, asociado a úlceras orales y adenopatías submaxilares. Los exámenes de laboratorio no evidencian neutropenia ni infección por virus respiratorios habituales. La serología para VEB (IgM VCA), la antigenemia para CMV y los cultivos faríngeo corriente y para hongos resultan negativos.

Se administra tratamiento antibiótico y antifúngico en forma empírica y se suspende MMF, sin obtener mejoría clínica. La paciente evoluciona con respiración bucal y ronquido nocturno, secundarios a aumento de volumen de la amígdala derecha, y dolor abdominal difuso. Dentro del estudio destaca: LDH aumentada $(505 \mathrm{U} / \mathrm{L})$, función renal, pruebas hepáticas, amilasa, lipasa y test de Widal normales. La ecografía abdominal evidencia numerosas lesiones focales hipoecogénicas en hígado y bazo; la tomografía axial computada (TAC) cervical confirma el aumento de volumen amigdalino derecho y múltiples adenopatías (Figura 1). Se descarta infección por Bartonella henselae por serología y Aspergillus por detección de antígeno galactomanana. Se repite serología para VEB que resulta negativa; pero la carga viral cuantitativa por reacción de polimerasa en cadena (PCR) en tiempo real resulta de 9.000 copias/mL de plasma. Se disminuye dosis de tacrolimus a la mitad y se realiza biopsia de amígdala y adenopatía cervical. El análisis de ambas muestras resulta negativo para bacterias, hongos, mycobacterias y virus herpes simplex; siendo positiva la PCR para VEB en tejido linfoide.

El análisis histológico muestra extensa proliferación linfoide atípica CD20 + e hibridación in situ para ARN VEB (EBER-1) positiva, hallazgos compatibles con TLPT de tipo polimorfo, inmunofenotipo B, asociado a infección por VEB (Figura 2). Se decide observar respuesta a disminución de inmunosupresión con seguimiento clínico e imagenológico. Evoluciona con disminución de úlceras orales y disfagia y regresión progresiva de lesiones hepatoesplénicas en ecografías seriadas. A los 10 días de modificación de la terapia la carga viral es de 260 copias/mL de plasma.

Debido al efecto antitumoral descrito en la literatura ${ }^{7,8}$ se decide cambiar el tratamiento inmunosupresor a sirolimus, MMF y prednisona $\mathrm{y}$ suspender tacrolimus. A diez meses de seguimien-
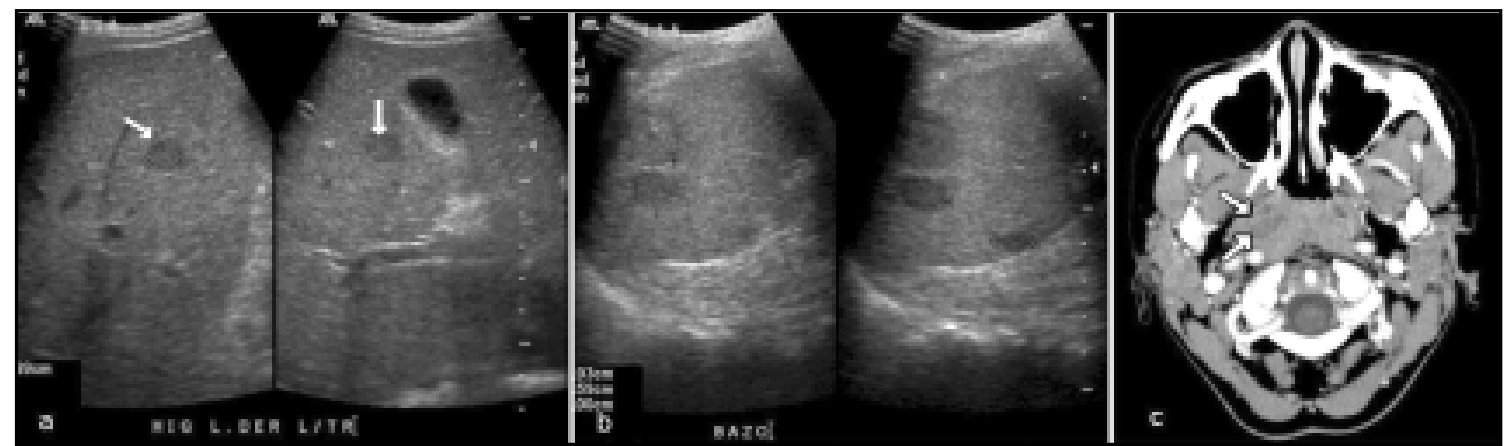

Figura 1. Ecotomografía abdominal. (a) Se observa una lesión focal hipoecogénica (flecha) de 1 cm de diámetro en lóbulo hepático derecho. (b) Otras lesiones de similares características son visibles en el bazo, de hasta 2,5 cm de diámetro. (c) TAC de cuello. Existe importante aumento de volumen de la amígdala derecha (flechas). 


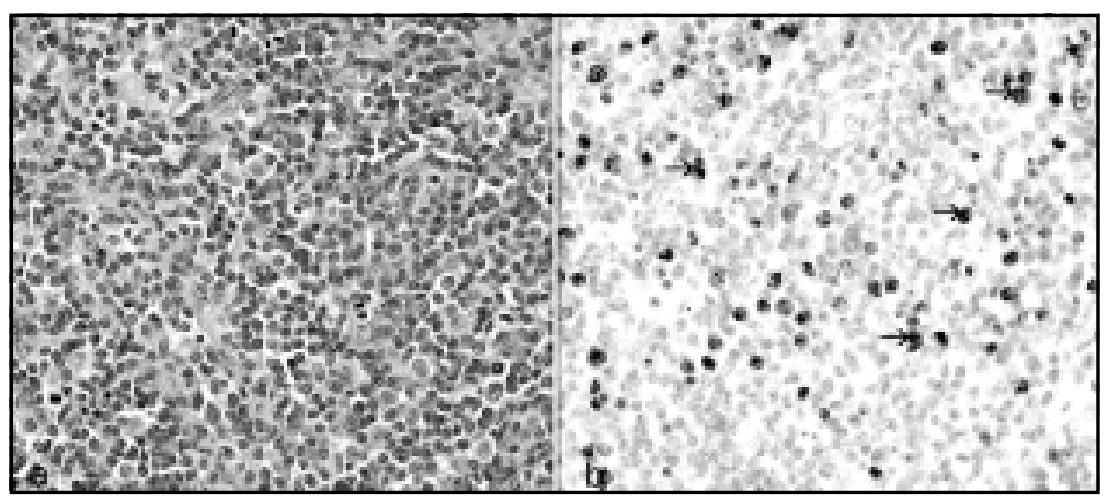

Figura 2. Biopsia de amígdala. (a) Microscopia de luz (x 400), HE. Extensa proliferación linfoide atípica. (b) Hibridización in situ para ARNs codificados por VEB (EBER-1); la sonda detecta señal positiva nuclear en las células linfoides atípicas (flechas).

to, la paciente se encuentra en excelentes condiciones clínicas, sin evidencias de rechazo y con desaparición de lesiones intraabdominales.

\section{DISCUSIÓN}

Los TLPT emergen en los años 90 como una complicación del trasplante de órganos sólidos ${ }^{4}$, asociada al desarrollo de inmunosupresores más potentes. Constituyen la neoplasia post trasplante más común en niños $(52 \%)^{9,10}$. La ausencia de una definición universalmente aceptada determina gran variación en la incidencia comunicada ${ }^{5}$, la cual depende además del órgano trasplantado, edad del receptor e inmunosupresión utilizada. Los trasplantes intratorácicos y los intestinales presentan las mayores tasas de TLPT (5\%-10\%), mientras que los renales alcanzan $2 \%-4 \%$ a $3-5$ años post trasplante $5,9,11$. Estas diferencias se deben a la cantidad de tejido linfoide de cada órgano y los esquemas de inmunosupresión utilizados. La incidencia de TLPT en pediatría es cuatro veces mayor que en adultos, dada la mayor proporción de niños seronegativos para VEB y con inmunosupresión más potente. La mortalidad por TLPT en niños con trasplante renal alcanza $48 \%$, siendo mayor que en receptores de otros órganos sólidos ${ }^{12}$.

La infección primaria por VEB es un factor de riesgo conocido de TLPT; los pacientes seronegativos con donante seropositivo presentan mayor riesgo de desarrollarlo ${ }^{13}$, como en el caso presentado. En receptores pediátricos hasta $90 \%$ se asocia a infección por VEB. Ésta puede provenir del órgano donado, del uso de productos sanguíneos $\mathrm{O}$ ser adquirida posteriormente ${ }^{14}$. El VEB permanece latente en el huésped tras la primoinfección, siendo capaz de transformar e inmortalizar a los linfocitos B, los cuales pueden proliferar en forma descontrolada. En el huésped inmunocompetente, dicha proliferación es limitada por los linfocitos T citotóxicos (LTC). En los pacientes trasplantados, la inmunosupresión limita la función linfocitaria, permitiendo la proliferación descontrolada de las células B infectadas ${ }^{13}$.

En general, a mayor inmunosupresión, mayor riesgo de TLPT. La contribución relativa de drogas específicas es controversial ${ }^{14}$. Un metanálisis comparó la incidencia de TLPT entre usuarios de ciclosporina y tacrolimus sin observar diferencias significativas ${ }^{15}$. El uso de MMF no se ha asociado a mayor riesgo. Estudios recientes sugieren menor incidencia de neoplasias con el uso de inhibidores de MTOR (mammalian target of rapamycin): sirolimus y everolimus, comparados con inhibidores de calcineurina ${ }^{7,8}$. Otros factores de riesgo descritos son la coinfección con CMV y hepatitis $\mathrm{C}^{9,16}$.

En los niños la mayoría de los casos de TLPT ocurre durante el primer año post transplante?; en el caso descrito el cuadro apareció al sexto mes de evolución. Los síntomas son múltiples y variados, desde un cuadro viral inespecífico hasta una proliferación monoclonal de células $\mathrm{B}$; puede 
confundirse con rechazo del injerto o asociarse a infecciones oportunistas ${ }^{9,12}$ (Tabla 1). El compromiso del injerto por TLPT ocurre hasta en un tercio de los trasplantes renales ${ }^{12}$. El estudio de laboratorio inicial debe incluir hemograma y evaluación de función renal y hepática; la elevación de ácido úrico y lactato deshidrogenasa son frecuentes. Las imágenes suelen demostrar enfermedad nodal o extranodal ${ }^{14}$. Otros estudios complementarios dependerán de cada paciente.

La serología de VEB y los tests monospots no son útiles para el diagnóstico de TLPT en pacientes trasplantados 5 . Existen diferentes métodos para la detección de ADN de VEB para el diagnóstico precoz y seguimiento de TLPT; la PCR cuantitativa en tiempo real es considerada actualmente como el método de elección. La muestra ideal en que debe medirse la carga viral es controversial: plasma, células mononucleares en sangre periférica o sangre total ${ }^{17}$; una carga viral de más de 10.000 copias/ml de plasma tendría una sensibilidad y especificidad de $100 \%$ para el diagnóstico de TLPT $^{18}$. Existe consenso en que los pacientes con TLPT tienen cargas virales significativamente mayores que los pacientes trasplantados sin TLPT, pero no está claro qué valores de corte son predictores de TLPT. Una carga viral en ascenso, más que un valor de corte único, sería más apropiado para identificar al paciente en riesgo ${ }^{17,19}$. En nuestra paciente, la serología fue negativa para VEB y la medición de carga viral confirmó la replicación de genes virales en sangre. La cuantificación inicial resultó cercana al valor diagnóstico reportado en la literatura, evidenciándose un descenso posterior al comienzo de la terapia.

El diagnóstico definitivo es histopatológico, clasificándose en 3 categorías: a) hiperplasia benigna, con proliferación difusa de células mononucleares y arquitectura tisular conservada; b) TLPT polimórfico, con linfocitos en distintas etapas de diferenciación, destrucción de la arquitectura tisular y áreas de necrosis y c) TLPT monomórfico, con transformación neoplásica del tejido. La mayoría de los TLPT deriva de células B, identificables con tinción para CD20. La hibridización in situ con sonda EBER-1 (Epstein Barr early RNA), identifica ARN de VEB en células infectadas. La biopsia de nuestra paciente fue compatible con TLPT polimórfico de estirpe B e infección por VEB.

No existen estudios que en forma aleatoria evalúen la eficacia de los distintos tratamientos, los cuales están dirigidos a aumentar la respuesta linfocitaria $\mathrm{T}$, reducir la proliferación de células $\mathrm{B}$, uso de citoquinas e inhibidores de replicación y diseminación viral (Tabla 2) 5,9,14,16. La reducción de la inmunosupresión constituye la primera línea de tratamiento, sin existir actualmente un esquema de consenso. Es fundamental el seguimiento

Tabla 1. Elementos clínicos de T LPT

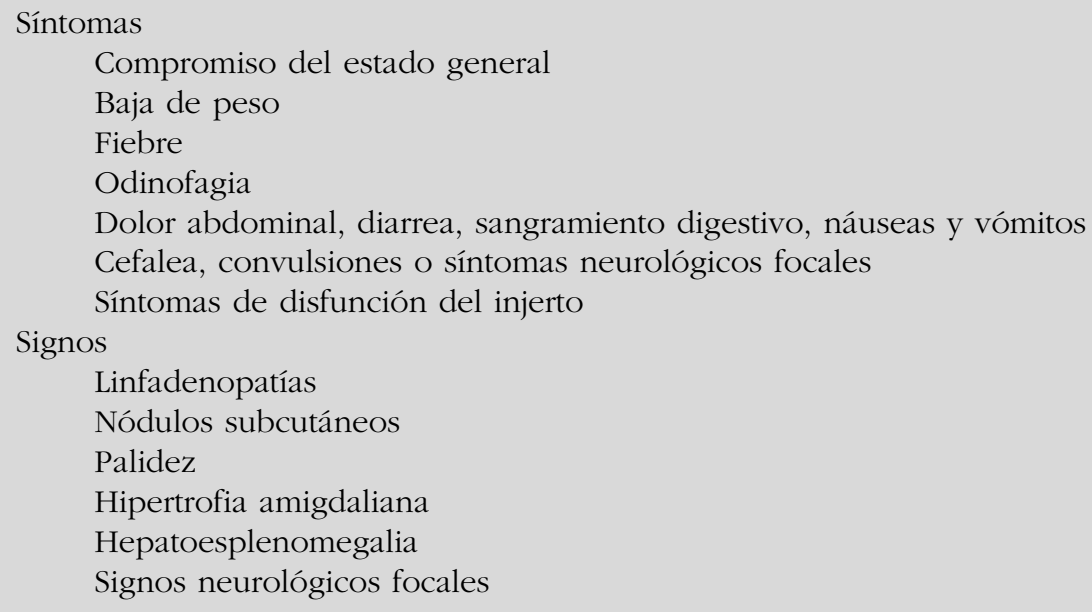


Tabla 2. Estrategias de tratamiento en T LPT

Terapias Comentarios

\section{Primera línea}

Reducción de inmunosupresión

Terapia antiviral (Ganciclovir)

Quimioterapia

\section{Segunda línea}

Interferon alfa

Inmunoglobulinas endovenosas

Anticuerpos monoclonales anti células B

Generación de linfocitos T citotóxicos

específicos contra VEB

Quimioterapia

Cirugía (con o sin radioterapia)

\section{Nuevas terapias}

Basadas en células dendríticas

Inhibidores TOR (Sirolimus, everolimus)

Bexaroteno
Efectivo en la mayoría de los pacientes pediátricos, especialmente en PTLD polimórfico. Ampliamente utilizado, pero no probado Uso en caso de malignidad

Puede causar rechazo agudo

No probado, estudios en prevención

Resultados prometedores en enfermedad polimórfica refractaria.

Mínima experiencia en trasplante de órganos sólidos.

Uso en PTLD refractario o en recaída, más frecuente en adultos, PTLD monomórfico y enfermedad de inicio tardío.

Uso en compresión local de estructuras críticas, obstrucción intestinal, biopsia excisional de lesiones de fácil acceso.

En evaluación del paciente para determinar nuevas intervenciones. El uso de antivirales es controversial; éstos inhiben el ciclo lítico replicativo del VEB, pero no afectan los virus latentes u oncogénicos presentes en los TLPT. Los anticuerpos monoclonales anti CD20 (Rituximab) están aceptados como terapia de segunda línea ${ }^{9,20}$. Para lesiones más graves o sin respuesta a tratamiento, se utilizan protocolos de quimioterapia para linfoma no Hodgkin. En la actualidad existe interés en el rol de los inhibidores de mTOR por sus propiedades inmunosupresoras y antiproliferativas ${ }^{7-9}$. En este caso se redujo la inmunosupresión y se evaluó clínicamente y con ecografías seriadas a la paciente, la que presentó una regresión completa de la enfermedad. Posteriormente se cambió el tratamiento inmunosupresor a sirolimus, manteniendo función renal estable.

La prevención es fundamental para reducir la incidencia de TLPT. Actualmente es posible monitorizar pacientes de alto riesgo mediante medición seriada de carga de ADN de VEB mediante PCR o efectuar profilaxis con antivirales, cuya eficacia es controversial $^{5,17}$. Sin embargo, la primera estrategia debería ser la inmunización contra VEB antes de la inmunosupresión; existiendo varias vacunas, pero aún en fase de evaluación.

\section{Agradecimientos}

Los autores agradecen al Dr. Carlos Toledo R. por facilitar el material radiológico y contribuir a la interpretación de las imágenes. 


\section{REFERENCIAS}

1. Abecassis M, Barlett ST, Collins AJ, Davis CL, DelmoniCo FL, Friedewald JJ ET al. Kidney transplantation as primary therapy for end-stage renal disease: a National Kidney Foundation/Kidney Disease Outcomes Quality Initiative (NKF/KDOQITM) conference. Clin J Am Soc Nephrol 2008; 3: 471-80.

2. Wolfe RA, Ashby VB, Milford EL, Ojo AO, Ettenger RE, AgOdoA LYC ET AL. Comparison of mortality in all patients on dialysis, patients on dialysis awaiting transplantation, and recipients of a first cadaveric transplant. $N$ Engl J Med 1999; 341: 1730-35.

3. Dantal J, Pohanka E. Malignancies in renal transplantation: un unmet medical need. Nephrol Dial Transplant 2007; 22 [Suppl 1]: i4-i10.

4. PENN I. The problem of cancer in organ transplant recipients: An overview. Transplant Sci 1994; 4: 23-32.

5. Dharnidharka VR, Araya C. Post-transplant lymphoproliferative disease. Pediatr Nephrol 2007; DOI 10.10007/s00467-007-0582-3. Disponible en www.springerlink.com [Consultado el 15 de Febrero de 2008].

6. NALESNIK MA. The diverse pathology of post-transplant lymphoproliferative disorders: the importance of a standardized approach. Transpl Infect Dis 2001; 3: 88-96.

7. Pascual J. Post transplant lymphoproliferative disorder - the potencial of proliferation signal inhibitors. Nephrol Dial Transplant 2007; 22 [Suppl 1]: 127-35.

8. Gutiérrez Dalmau A, Campistol JM. The role of proliferation signal inhibitors in post-transplant malignancies. Nephrol Dial Transplant 2007; 22 [Suppl 1]: i11-i16.

9. Shroff R, Rees L. Post transplant lymphoproliferative disorder- a literature review. Pediatr Nephrol 2004; 19: 369-77.

10. Collins MH, Montone KT, Leahey AM, Hodinka RL, Salhany KE, Kramer DL el al. Post-transplant lymphoproliferative disease in children. Pediatr Transplantation 2001; 5: 250-7.

11. Libertiny G, Watson CJ, Gray DW, Welsh Ki, Morris PJ. Rising incidence of post transplant lymphoproli- ferative disease in kidney transplant recipients. $B r J$ Surg 2001; 88: 1330-4.

12. Holmes RD, Sokol RJ. Epstein Barr virus and post transplant lymphoproliferative disease. Pediatr Transplantation 2002; 6: 456-64.

13. Allen Ud, Farkas G, Hébert D, Weitzman S, Stephens D, Petric M El Al. Risk factors for post-transplant lymphoproliferative disorder in pediatric patients: A case control study. Pediatr Transplantation 2005; 9: 450-5.

14. Green M, Webber S. Posttransplantation lymphoproliferative disorders. Pediatr Clin N Am 2003; 50: 147191.

15. Webster aC, Woodroffe RC, Taylor RS, Chapman JR, Craig JC. Tacrolimus versus ciclosporin as primary immunosupression for kidney transplant recipients: meta-analysis and metaregression of randomized trial data. BMJ 2005; 331: 810.

16. Lim WH, Russ GR, Coates P. Review of Epstein Barr virus and post transplant lymphoproliferative disorder post-solid organ transplantation. Nephrology 2006; 11: 355-66.

17. Bakker N, Van Imhoff G, Verschuuren E, Van Son W. Presentation and early detection of post-transplant lymhoproliferative disorder after solid organ transplantation. Transplant International 2007; 20: 207-18.

18. Wagner HJ, Wessel M, Jabs W, Smets F, Fischer L, OfFner G ET AL. Patiens at risk for development of posttransplant lymphoproliferative disorder: plasma versus peripheral blood mononuclear cells as material for quantification of Epstein Barr viral load by using real-time quantitative polymerase chain reaction. Transplantation 2001; 72: 1012-19.

19. Tsai D, Nearey M, Hardy CL, Tomaszewski Je, Kotloff RM, Grossman RA ET al. Use of EBV PCR for the diagnosis and monitoring of post transplant lymphoproliferative disorder en adult solid organ transplant patients. Am J Transplant 2002; 2. 946-54.

20. Oertel SH, Verschuuren E, Reinke P, Zeidler K, PappVary M, Babel N et al. Effect of Anti-CD 20 Antibody Rituximab in Patients with Post-Transplant Lymphoproliferative Disorder (PTLD). Am J Transplant 2005; 5: 2901-6. 